\title{
Centennial to millennial geomagnetic field variations
}

\author{
Monika Korte ${ }^{1, *}$ and Raimund Muscheler ${ }^{2}$ \\ 1 Helmholtz-Zentrum Potsdam, Deutsches GeoForschungsZentrum GFZ, Telegrafenberg, 14473 Potsdam, Germany \\ *corresponding author: e-mail: monika@gfz-potsdam.de \\ 2 Department of Geology, Lund University, Sölvegatan 12, SE-22362 Lund, Sweden
}

Received 27 February 2012 / Accepted 7 June 2012

\begin{abstract}
Reconstructions of the geomagnetic field in the past represent a useful tool not only to investigate the geodynamo process, but also to estimate the effect of geomagnetic shielding for any studies on cosmogenic radionuclides and galactic cosmic rays. A number of new millennial-scale geomagnetic field reconstructions have been published over the last years, based on improved global archeo- and paleomagnetic data compilations. Here we review several spherical harmonic models and compare their dipole field predictions to reconstructions based on virtual axial dipole moments and virtual geomagnetic poles. Dipole intensity estimates from cosmogenic radionuclide production records, with suitable filtering to minimise the solar influence, have also been included in the comparison to provide independent information about variations in the strength of the geomagnetic field. However, due to differences among geomagnetic models and between ${ }^{14} \mathrm{C}$ and ${ }^{10} \mathrm{Be}$ production records this comparison is fairly inconclusive with respect to multicentennial variations. Different geomagnetic dipole tilt reconstructions agree well for much of the Holocene, but dipole moment estimates still differ substantially. Recent spherical harmonic models for the past 3 and 10 kyrs have improved considerably compared to earlier versions. Nevertheless at present we recommend to test if any interpretation depends on the choice of model.
\end{abstract}

Key words. magnetic field - geomagnetic dipole intensity - geomagnetic shielding - cosmogenic radionuclide production

\section{Introduction}

Knowledge about past geomagnetic field variations on centennial to millennial scales is not only important to gain better understanding of the geodynamo process in the Earth's core, but also to estimate the geomagnetic shielding effect against galactic cosmic rays in space climate studies. This is essential when using radionuclide production rates to infer past solar activity, which is of high interest to reveal the solar influence on Earth's climate variability (e.g., Marsh \& Svensmark 2003; Solanki et al. 2004; Muscheler et al. 2005b; Usoskin et al. 2006; Snowball et al. 2007). Other applications of past geomagnetic field reconstructions include investigations of atmospheric ionisation by galactic cosmic rays (e.g., Usoskin et al. 2008, 2010) or in-situ cosmogenic nuclide production rates to study Earth surface processes (e.g., Pigati \& Lifton 2004; Lifton et al. 2008).

Information about the magnetic field evolution prior to the times of direct observations is determined in laboratory procedures from archeo- and paleomagnetic material. Substantial progress has been made over the past 10 years to compile all published Holocene results (Korte et al. 2005; Genevey et al. 2008; Korhonen et al. 2008; Donadini et al. 2009). These global compilations have been used to develop global field models, either describing only the dipole evolution (Genevey et al. 2008; Knudsen et al. 2008; Valet et al. 2008; Nilsson et al. 2010) or also including non-dipole contributions (Korte \& Constable 2005a, 2011; Korte et al. 2009, 2011). The variety of models can be confusing, particularly as some substantial differences among them remain.

Cosmogenic radionuclide production rates can also be used to gain independent information about past geomagnetic field changes, if the modulations by the solar magnetic field can be eliminated or ignored. On short time scales of decades to a few centuries this is not possible, as the solar influence clearly dominates the production signal (e.g., Snowball \& Muscheler 2007) and local climate can also play a role (e.g., Usoskin et al. 2009). However, multi-millennial variations show clear resemblance to geomagnetic field changes. Although there is no direct way to separate solar from geomagnetic influences in radionuclide production records, it is instructive to compare their variations to geomagnetic field reconstructions.

Here, we aim to summarise present knowledge about the Holocene magnetic field evolution and remaining uncertainties, mainly concentrating on the dipole contribution and only briefly mentioning progress in describing regional differences in field intensity. We first discuss important archeo- and paleomagnetic data characteristics. We then review recently developed millennial-scale spherical harmonic geomagnetic field models and compare their dipole predictions to virtual axial dipole and virtual geomagnetic pole (VGP) reconstructions, discussing uncertainties in absolute values and variations. Finally, we include a comparison to past geomagnetic field estimates obtained with multi-centennial to millennial-scale filtering from ${ }^{14} \mathrm{C}$ and ${ }^{10} \mathrm{Be}$ radionuclide production records. We conclude with an outline of the important requirements to make further progress in obtaining reliable and robust reconstructions of the past geomagnetic field.

\section{Available magnetic data}

Knowledge about geomagnetic field directions and intensity prior to direct observations comes from archeo- and paleomagnetic data. A large number of such data have been produced over the past decades in paleomagnetic laboratories around 


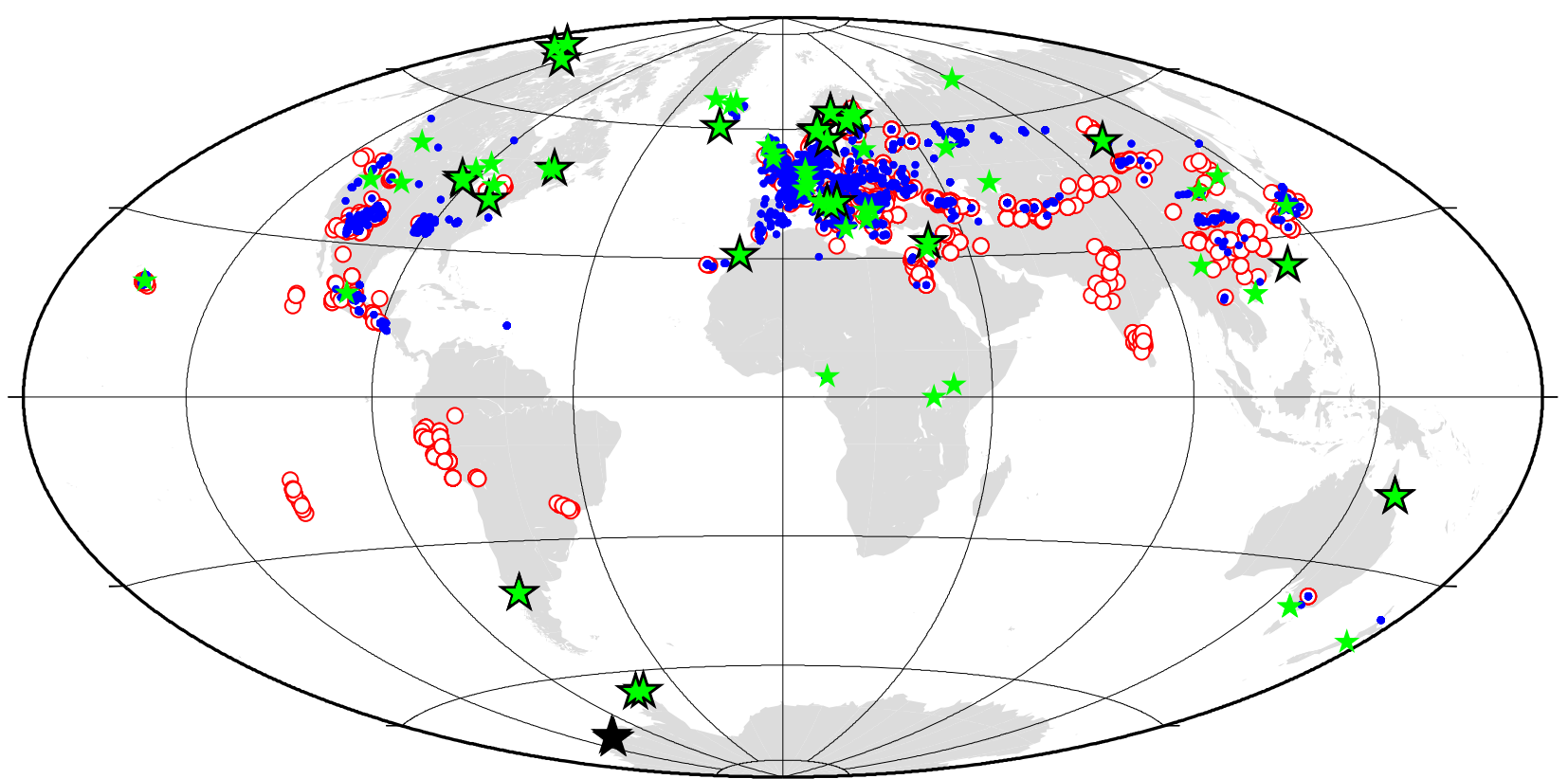

Fig. 1. Distribution of available Holocene archeo- and paleomagnetic data (status as described by Donadini et al. 2009 and Korte et al. 2011). Archeointensities (red circles) and particularly archeomagnetic directions (blue dots) are scarce in the southern hemisphere. Sediment relative intensity (black stars/black borders around green stars) and directional (green stars) records provide a somewhat better global distribution.

the world, so that the amount of available data is getting large enough to allow for global inversion models of the Holocene magnetic field. One of the latest, most comprehensive data collections is described by Donadini et al. (2009), to which 13 more sediment records have been added for more recent modelling work by Korte \& Constable (2011). The past field information is gained from a variety of materials through a range of different laboratory procedures (see, e.g., Donadini et al. 2010, for a recent review). Consequently, the accuracy and reliability of the data vary notably and can affect field reconstructions in different ways.

The data are broadly divided into two categories. Firstly, results come from material carrying a thermoremanent magnetisation that has been obtained by cooling through the Curie temperature of the included magnetic minerals. Archeomagnetic data obtained from burnt archeological artifacts, like pot-shards or bricks, but also data from lava flows fall in this category. In the following, the term "archeomagnetic" data therefore includes data from lavas. Secondly, results come from sediment cores that have acquired their magnetisation by embedding magnetic grains aligned with the ambient geomagnetic field. The two types of data have different characteristics, with both advantages and disadvantages for global modelling purposes.

Oriented archeomagnetic samples can provide information about field directions, and past field intensity can be determined in laboratory experiments. These experiments, however, often fail stringent quality checks and the uncertainties associated with paleointensity data are generally large (e.g., Donadini et al. 2010). Uncertainties in the ages of these kind of data should in general be no larger than those of the dating methods, as the natural cooling process during which the magnetisation is acquired is comparatively fast. In ideal cases they can be of the order of a few years through historical or archaeological dating, although they commonly lie in the range of some decades and can reach a few centuries. The disadvantages of archeomagnetic data for global field reconstruction are their scattered distribution in time and space and their extremely sparse coverage of the southern hemisphere (Fig. 1). The number of available data is relatively high for the past 2 kyrs, but drops dramatically for older times. A comprehensive global compilation of archeomagnetic data of the past 50 kyrs is provided by the GEOMAGIA.v2 database (Donadini et al. 2006; Korhonen et al. 2008) at http://geomagia.ucsd.edu/.

Sediment records in contrast offer long time series and their global distribution is somewhat better (Fig. 1), though also strongly biased towards Europe. They provide directional information, but declination sometimes is only known as relative variations. Measurements of the strength of magnetisation can only provide relative variations of past field intensity, and only if the changes can be suitably normalised in order not to reflect lithological or environmental changes. Methods to scale them to absolute intensities include comparison to globally averaged virtual axial dipole moments (VADM) or to nearby archeomagnetic intensity data.

Age information for sediment records is mostly provided by age-depth models which are constructed based on radiocarbon dated tie points and relative stratigraphic information. The radiocarbon method in itself, with the necessity for calibration to calendar ages, contains rather large uncertainties of several decades. The uncertainties can reach centuries or even millennia due to carbon reservoir effects, wrong assumptions about sedimentation rates between and beyond the tie-point ages and due to the "lock-in depth", which means that magnetic particles might remain subject to adjustment while the sediment is still unconsolidated. In that case the sediment age might be older than the age at which the magnetisation of the ambient field gets locked in. Some sediment cores show varves, which can be counted for relative age information. This can principally provide annual time resolution. However, missing or extremely fine layers may adulterate the results, and the experimental sample size also leads to magnetic results which are integrating over at least a couple of years. More details about sediment record characteristics and dating can be found, e.g., in the review by Donadini et al. (2010). 


\section{Spherical harmonic field reconstructions}

Continuous, spherical harmonic models of the geomagnetic field are convenient representations for many purposes. They provide predictions of any field component at any location on Earth and at any time within the validity interval of the model. They offer an easy way to distinguish between dominating dipole field and smaller scale non-dipole field contributions. Most attractively, they allow for upward and downward continuation within source-free regions, specifically to the top of the Earth's core and thus provide information about the geodynamo process.

The global field reconstructions of the CALSxk series (Continuous models based on Archeomagnetic and Lake Sediment data spanning the past $\mathrm{x}$ kyrs) are regularised spherical harmonic models. Continuity in time is provided by expanding the Gauss coefficients in cubic B splines. A summary of the modelling method and an overview over the whole CALSxk model series are given by Korte \& Constable (2011). Here, we mainly consider the most recent models CALS10k.1b and CALS3k.4, and only summarise the modelling aspects which influence the discussed results. For comparison we include CALS7K.2 and CALS3k.3, which have been widely used before.

An important aspect common to all the CALSxk models is the regularisation in space and time. The degree of the spherical harmonic expansion and the knot-point spacing of the splines are chosen to be higher than the spatial and temporal resolution expected from the data. Regularisation factors are then used to trade off fit to the data against smoothing constraints, which in the extreme case would give a pure dipole model and linear time evolution. The choice of the damping factors determines the spatial and temporal resolution of a model. Ideally, they are chosen such that the data are fitted just within their uncertainty estimates, so that the amount of structure shown by the model is the minimum structure required by the data. Unfortunately, data and age uncertainties in the case of archeo- and paleomagnetic data are not well determined, and the age uncertainties cannot be taken into account properly in the inversion scheme. The criterion to choose the regularisation factors, therefore, has been based on a comparison to geomagnetic power spectra of the current and historical main field and secular variations. The presented models display comparable power in low spherical harmonic degrees with strong drop of power for higher degrees (see, e.g., Fig. 10 of Korte et al. 2009), but it is clear that the choice of regularisation factors remains somewhat subjective and a model is far from a unique solution to the data inversion.

Model CALS10k.1b (Korte et al. 2011) covers the time interval from $8000 \mathrm{BC}$ to $1990 \mathrm{AD}$. It is dominated by sediment data. Relative sediment intensity data have been included by first scaling them using model CALS3k.3 and then applying a re-scaling together with outlier rejection in four model iterations. As it had turned out that even large-scale features of the model are rather sensitive to changes in the data set, particularly for the earlier part the model, the final model was obtained as the average of 2000 models where data and ages were varied within their uncertainty estimates and bootstraps on the final data sets were performed (hence version number 1b). Model uncertainty estimates are provided as one standard deviation from this averaging. The averaging has a strong effect on the spatial and temporal resolution of the model. Octupole and higher contributions are damped and the temporal resolution is no better than about 500 years. The historical to recent centuries of the model are strongly constrained to agree with the 400-year historical model gufm1 by Jackson et al. (2000), and consequently spatial and temporal resolution increase drastically for this time interval.

Model CALS3k.4 (Korte \& Constable 2011) spans the time $1000 \mathrm{BC}$ to $1990 \mathrm{AD}$ and is based on the same modelling strategy and data set as CALS10k.1b, except for the bootstrap averaging. It has a higher effective resolution on the order of spherical harmonic degree 5 and around 100 years in time.

Model CALS3k.3 (Korte et al. 2009) is the predecessor of CALS3k.4, spanning the same time interval. The data basis is smaller by 13 sediment records, mainly influencing the regions about South-East Asia, Alaska and Siberia. Relative intensity records scaled by archeomagnetic data and a model purely from such data have been used, but without iterative re-scaling. The agreement with gufml at the historical end is weaker.

Model CALS7K.2 (Korte \& Constable 2005a) covers the interval $5000 \mathrm{BC}$ to $1950 \mathrm{AD}$. As the $10 \mathrm{kyr}$ model, it is dominated by sediment data prior to $1000 \mathrm{BC}$. The data basis is smaller than that for CALS10k.1b by 33 sediment records and approximately 2500 archeomagnetic data points. Most importantly, no relative intensity records were used, so that field intensity particularly in the early millennia was determined by very few archeomagnetic intensity data only. No bootstrapping has been performed and, in this case, the historical to recent part is not constrained in any way. This model clearly is outdated now.

All models are available together with some Fortran source code to obtain model predictions and coefficients from the EarthRef Digital Archive http://www.earthref.org. Model predictions can also be obtained interactively from the GEOMAGIA.v2 database at http:/geomagia.ucsd.edu/.

The spherical harmonic models do not only provide estimates of the past dipole evolution, as will be discussed in the next section, but also give information about regional differences in magnetic field strength. At present day, it is well known that magnetic shielding is weak in the southern Atlantic region, the so-called South Atlantic Anomaly (SAA), with important implications, e.g., for space weather influences on spacecraft (Heirtzler 2002). Figure 2a shows that the SAA can be clearly recognised in an average of field intensity at the Earth's surface from 1590 to 1990 as predicted by the gufm1 model by Jackson et al. (2000). Averages over 3, 7 and 10 kyrs from the CALSxk models (Figs. 2b-2d) suggest that, although there are zonal differences of minimum field intensity, the southern Atlantic is no preferred location for the minimum field strength. Much of the time the minimum field strength appears to have lain further east, towards the SouthEast Asian region. This result could be of interest to estimate regional differences in past magnetic field shielding. However, it should be taken with some reservation, as no intensity data were available south of $30^{\circ} \mathrm{N}$ for the longitudinal band between $45^{\circ} \mathrm{W}$ and $120^{\circ} \mathrm{E}$ for any of the millennial-scale models. African intensity data or independent estimates of regional differences in magnetic shielding are required to confirm or revise these observations.

\section{Geomagnetic dipole reconstructions}

We now summarise present knowledge about the past dipole and dipole tilt evolution. Alternative reconstructions of the past dipole evolution, that can be obtained more easily from less comprehensive data collections, are based on VADM and 


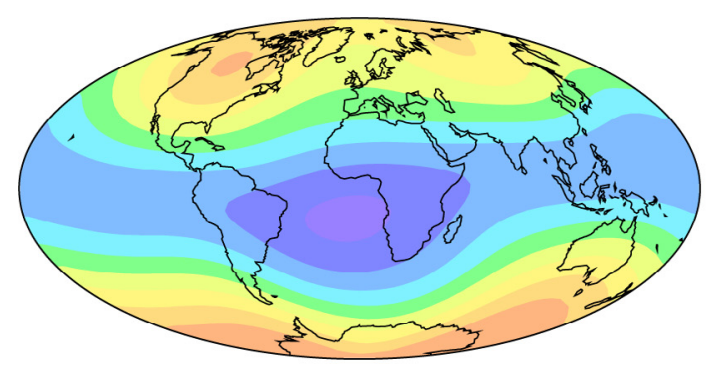

(a) gufm1 1590AD-1990AD

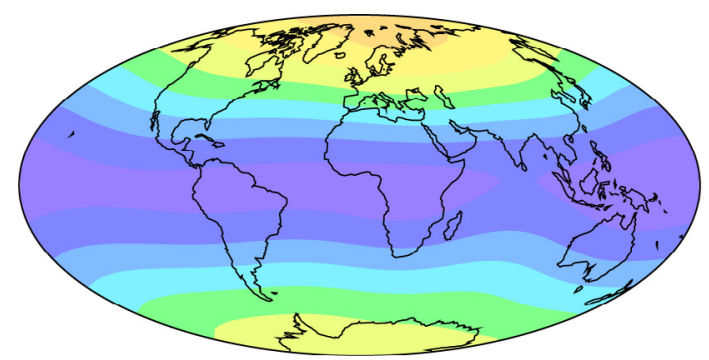

(c) CALS7K.2 5000BC-1950AD
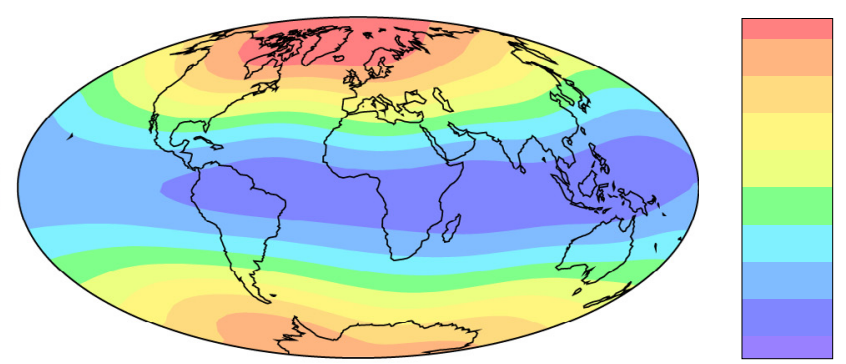

$\mu \mathrm{T}$

70

60

50

40

(b) CALS3k.4 1000BC-1990AD
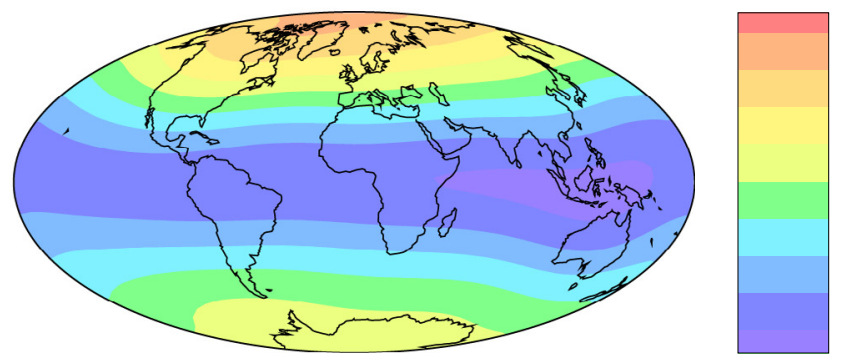

$\mu \mathrm{T}$

70

Fig. 2. Field intensity distribution at the Earth surface predicted by spherical harmonic field models averaged over 400 years (a) model gufm1, 3 kyrs (b) model CALS3k.4, 7 kyrs (c), outdated model CALS7K.2 and 10 kyrs (d) model CALS10k.1b.

VGP. VADMs are obtained by translating archeomagnetic intensity results to the strength of a pure axial dipole field with that intensity at the given geographic latitudes. Similarly, VGPs are obtained as the poles of a pure, but tilted, dipole field from magnetic directional results. At first glance they seem more robust than spherical harmonic models, which have more degrees of freedom so that inconsistent data might lead to some dispersion of large-scale power towards higher spherical harmonic degrees. However, VADM or VGP reconstructions rely on the assumption that the non-dipole field contributions average out if data are averaged in space and/or in time. Tests with present-day models show that the assumption is valid if averages can be obtained from a very good global data coverage, even without temporal averaging. Inhomogeneous data coverage on the other hand can bias the averages considerably (Korte \& Constable 2005b), and it is not clear what length of temporal averaging is necessary to alleviate or eliminate such biases. The comparisons of different models predicting past dipole moment and dipole axis orientation in Figures 3 and 4 highlight recent progress and remaining uncertainties in the different reconstructions.

Recent VADM reconstructions for the Holocene have been presented by Genevey et al. (2008) and Knudsen et al. (2008). Both also investigated the effect of regional biases in VADMs by binning the data regionally in different ways. While Genevey et al. (2008) conclude that regional weighting can improve VADM results from the presently available data for the past 3 kyrs and acknowledge that, due to the lack of southern hemisphere data, VADM results might be geographically biased towards western Eurasia, Knudsen et al. (2008) consider the differences they find over the last 10 kyrs insignificantly small. The "global" VADM results by Genevey et al. (2008) using equally weighted archeointensity averages from three to eight regional bins and sliding windows of 200 years shifted by 100 years are included in Figure 3 with one standard error

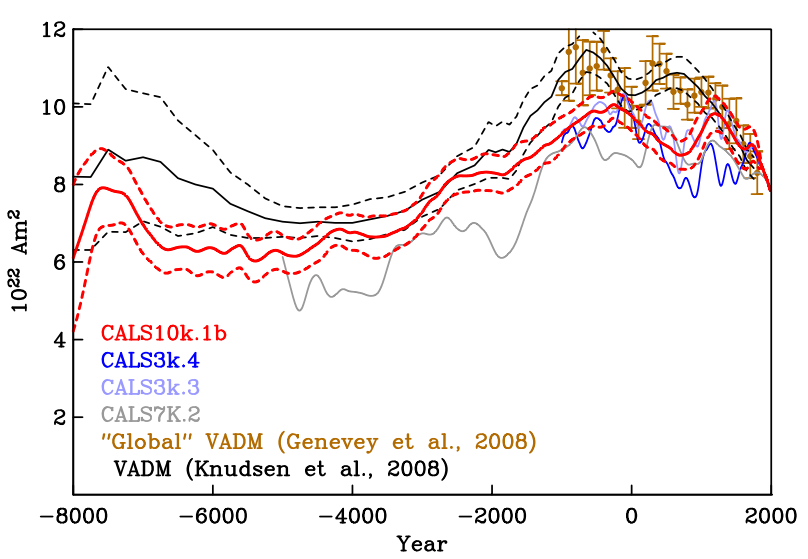

Fig. 3. Dipole moment predicted by spherical harmonic field models CALS10k.1b (red), CALS3k.4 (blue), CALS3k.3 (light blue), CALS7K.2 (grey) and by the archeomagnetic VADM reconstructions by Knudsen et al. (2008) (black) and Genevey et al. (2008) (brown) with uncertainty estimates (dashed lines and error bars) as provided by the authors, see text for details. Uncertainty estimates for CALS3k.3 and CALS3k.4, which are similar to those for CALS10k.1b, have been omitted to avoid cluttering the figure.

bar. The VADM reconstruction by Knudsen et al. (2008) is also included in Figure 3. It uses sliding window averages of width 500 years shifted by 100 years back to $2000 \mathrm{BC}$ and $1 \mathrm{kyr}$ shifted by 500 years back to $10000 \mathrm{BC}$. The two standard deviation uncertainty estimates shown in the figure are obtained from bootstrap re-sampling.

All dipole moment reconstructions show the well-established pattern of higher values during the recent $3 \mathrm{kyrs}$ and lower values before. The higher resolution dipole variations predicted by CALS3k.3 fall nicely within the one standard deviation bootstrap uncertainty estimates of the strongly 

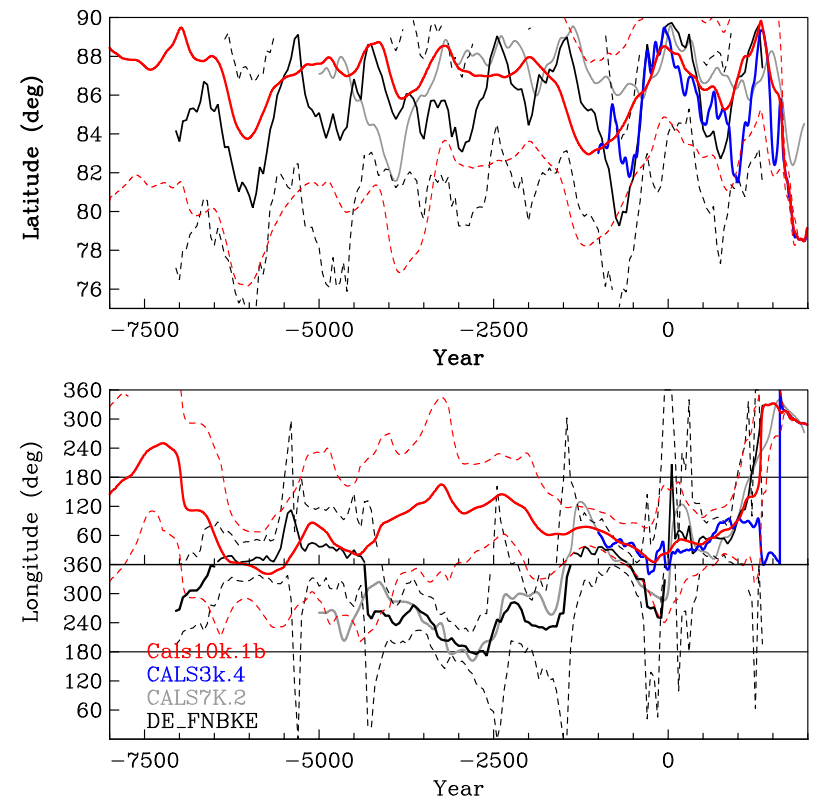

Fig. 4. Latitude (top) and longitude (bottom) of the northern geomagnetic pole location represent tilt and tilt direction of the magnetic dipole axis. Estimates are from spherical harmonic models CALS10k.1b (red with bootstrap uncertainty estimates as dashed lines), CALS3k.4 (blue), CALS7K.2 (grey) and the VGP reconstruction $\mathrm{DE}_{\mathrm{FNBKE}}$ (black with uncertainty estimates as dashed lines).

smoothed CALS10k.1b, but somewhat surprisingly the estimate by CALS3k.4 (and similarly of a strongly smoothed CALS3k.4b, see Korte \& Constable 2011) is lower during 0 to $1500 \mathrm{AD}$, although they are based on the same data sets. This is likely a consequence of the iterative re-scaling of the relative intensity records of different length and indicates that further investigations on quality and optimal scaling of relative intensity records are necessary to improve global field reconstructions. The fact that CALS7K.2 gives a lower dipole moment most of the time prior to $1000 \mathrm{BC}$ must be attributed to the lack of intensity information in this model during those times. In principle, with a perfect distribution of exact directional data, few intensity data should be enough to act as a scaling factor (Hulot et al. 1997). However, with the available real data there is a trade-off between fit to directional and fit to intensity data, which together with the influence of regularisation can lead to an underestimation of the field and dipole strength. In fact, the oldest part of CALS10k.1b, between 8000 and $7500 \mathrm{BC}$, is supported by very few intensity data and should be regarded with some caution for this reason. Spherical harmonic and VADM reconstructions agree, perhaps surprisingly, well between 4000 and $1500 \mathrm{BC}$, around $0 \mathrm{AD}$ and after $1000 \mathrm{AD}$. In general, however, the VADM results tend to be higher than the spherical harmonic estimates, which could be consistent with VADMs that are biased towards Eurasia (Korte \& Constable 2005b) for recent times. The strongest discrepancies occur around $500 \mathrm{BC}$ and 500 AD. Nilsson et al. (2011) computed a synthetic tilt modulated VADM record for a location in western Eurasia based on dipole strength estimates obtained from ${ }^{10} \mathrm{Be}$ flux and taking into account the dipole tilt based on the reconstruction of the northern geomagnetic pole position from VGP of globally distributed sediment records. They compared this synthetic VADM curve to the western Eurasia VADM curve by Genevey et al. (2008) and conclude from the mutual consistency but differing amplitudes that also the Knudsen et al. (2008) VADM reconstruction is probably influenced by geographically biased data towards western Eurasia during the recent 4 kyrs. However, we might have to wait for a good distribution of southern hemisphere archeomagnetic data to definitely settle the question whether VADMs give a regionally biased overestimate or spherical harmonic models underestimate the dipole moment during these times.

A dipole tilt model based on VGPs of five globally well-distributed and high-quality lake sediment records has been constructed by Nilsson et al. (2010) for the time period $7000 \mathrm{BC}$ to $1400 \mathrm{AD}$. Nilsson et al. (2011) discuss a potential $1350 \mathrm{yr}$ cyclicity from the nearly periodic variation of dipole tilt they observe in the slightly updated model $\mathrm{DE}_{\mathrm{FNBKE}}$. A comparison of dipole tilt reconstructions is shown as latitude and longitude of the northern hemisphere geomagnetic axis in Figure 4. First of all it is obvious that the uncertainty estimates associated with the tilt of the axis in all models are on the order of the dipole tilt angle itself. Nevertheless, the spherical harmonic and VGP reconstructions agree well in predicting a comparatively strong tilt between 6500 and $5500 \mathrm{BC}$ and in the variations predicted from about $1000 \mathrm{BC}$ to present. The present-day dipole tilt might seem exceptionally large particularly compared to the spherical harmonic model predictions. It is well possible that the amplitudes of tilt are damped by the limited temporal resolution of the models. The dipole axis longitude is not well defined when the axis is close to the geographic pole, therefore apparently abrupt longitudinal changes can occur when the tilt is very low and uncertainty estimates in longitude can be large. Note that there was an error in calculating these for Figure 2 of Korte et al. (2011) and the estimates shown here are corrected. Reasonable agreement among the models is seen much of the time, although they disagree relatively often in the direction of the axis movement. This is most apparent for the time interval from about 4500 to $1500 \mathrm{BC}$ :

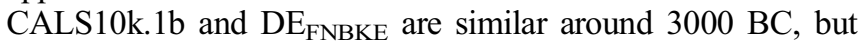
show opposite westward or eastward axis movements before and afterwards. It is interesting to note that the old CALS7K.2, based on much less data than CALS10k.1b, agrees closely with $\mathrm{DE}_{\mathrm{FNBKE}}$ in terms of axis longitude during these times, but rather resembles the predictions of CALS10k.1b regarding the axis tilt. It remains to be investigated further whether CALS10k.1b is adversely affected by some incompatible data, or whether the VGP reconstruction might suffer from a lack of data during this time interval.

\section{Cosmogenic radionuclide dipole estimates}

An alternative recorder for past geomagnetic field changes are cosmogenic radionuclide production rates, which are modulated by variations of both the solar magnetic field strength and the geomagnetic field intensity. Short wavelength magnetic field features deteriorate faster with distance from the source than long-wavelength fields. It is therefore generally assumed that geomagnetic modulation in ${ }^{14} \mathrm{C}$ or ${ }^{10} \mathrm{Be}$ records is related only to the dipole field contribution and not influenced by regional geomagnetic field differences due to the altitude at which the cosmic ray modulation occurs. However, regional differences in the radionuclide production rates are caused by the nature of dipole field. Cosmic rays do not get deflected at the poles due to the fact that the geometry of the geomagnetic field lines allows direct access of cosmic rays in these areas. By contrast, maximum deflection occurs at the equator. The regional differences in the radionuclide production rates are removed by 
atmospheric mixing of ${ }^{14} \mathrm{C}$. The degree of mixing is disputed for ${ }^{10} \mathrm{Be}$ but there is good evidence that ${ }^{10} \mathrm{Be}$ gets also well mixed and that, e.g., the signal measured in polar ice cores reflects mainly the global average production signal (see, e.g., Muscheler \& Heikkilä 2011, and references therein).

Therefore, cosmogenic radionuclide records can provide independent information about past dipole moment changes, but it is necessary to eliminate the influence of solar variations. Without additional information this is not really possible but, in general, it is assumed that short-term changes up to some centuries are dominated by the solar influence, and longer-term variations are dominated by the geomagnetic shielding (Snowball et al. 2007). For comparison purposes, we base our calculations on the assumption that the variations seen in ${ }^{14} \mathrm{C}$ and ${ }^{10} \mathrm{Be}$ records after applying different low-pass filters originate only from geomagnetic dipole variations. We are well aware of the fact that especially short-term changes appearing in the cosmogenic radionuclide-based geomagnetic field reconstructions could as well be due to solar variations.

For the following calculations we use updated versions of the radionuclide records presented in more detail in Muscheler et al. (2004, 2005a). A C production record has been calculated from the Intcal04 C calibration curve (Reimer et al. 2004). This calculation corrects for the known influences of the carbon cycle on the atmospheric $\mathrm{C}$ concentration (for details see Muscheler et al. 2005a). The $10 \mathrm{Be}$ record is a mean of the Be data from the GRIP and GISP2 ice cores from Summit in Central Greenland (Finkel \& Nishiizumi 1997; Muscheler et al. 2004; Vonmoos et al. 2006). We use the 10322 Be flux as the best proxy for past ${ }^{10} \mathrm{Be}$ production rate changes. However, the relative variations of the $\mathrm{Be}$ concentrations and the $\mathrm{Be}$ fluxes are very similar during the climatically stable Holocene period. We updated the Be flux with the most recent ice core time scale (GICC05) (Rasmussen et al. 2006) and the associated accumulation rates.

The cosmogenic radionuclide records have been low-pass filtered by a rectangular function in the frequency domain with different cut-off frequencies in order to minimise the solar influence and to investigate the time scales on which we see common changes in the radionuclide production rates and the geomagnetic field intensity reconstructions. Note that like any filtering this can lead to edge effects, i.e. in this case particularly to unreliable trends in the radionuclide-based records towards the youngest several hundred years of the data. The ${ }^{14} \mathrm{C}$ production record and the ${ }^{10} \mathrm{Be}$ flux records have been converted into geomagnetic field intensity estimates based on the results of Masarik \& Beer (1999) after the low-pass filtering. We normalised the cosmogenic radionuclide records in order to obtain the same average geomagnetic field intensity as indicated by the CALSxk.x models for the studied time intervals.

Figure 5 shows comparisons of the $1 / 1000 \mathrm{yr}$ (a) and $1 / 3000 \mathrm{yr}$ (b) low-pass filtered radionuclide records to the dipole moment reconstructions of CALS10k.1b and the VADMs by Knudsen et al. (2008). It is visible that the longterm trends (Fig. 5b) agree generally well. The ${ }^{10} \mathrm{Be}$ reconstruction exhibits larger multi-millennial variations than seen in CALS10k.1b. This could point to a dampening of the geomagnetic field intensity reconstruction in CALS10K.1b but it could as well be due to undetected climatic influences on the ${ }^{10} \mathrm{Be}$ flux. The ${ }^{14} \mathrm{C}$ curve shows an apparent phase shift during the past $5 \mathrm{kyr}$ compared to all other reconstructions, which could be attributed to relatively small long-term changes in the carbon cycle during the Holocene (Muscheler et al. 2004) or that our
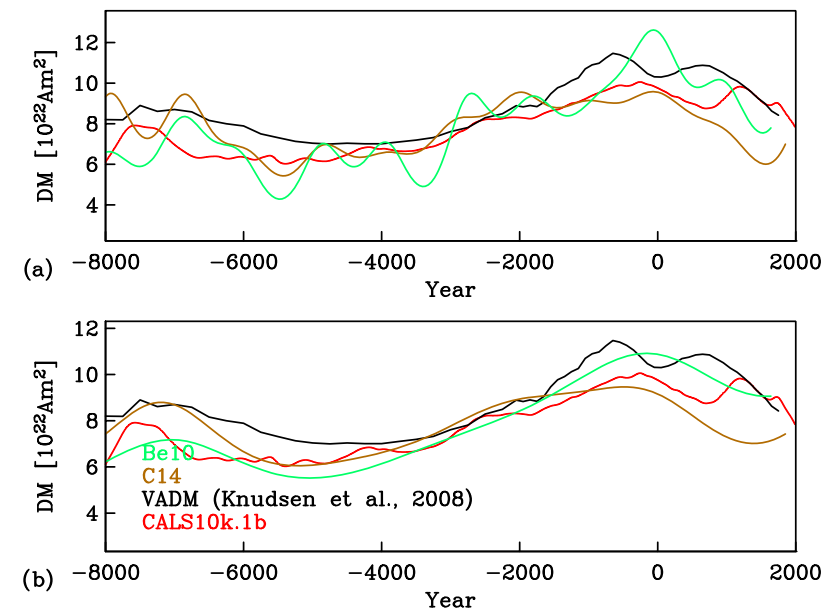

Fig. 5. Dipole moment estimates based on ${ }^{14} \mathrm{C}$ (brown) and ${ }^{10} \mathrm{Be}$ (green), low-pass filtered by $1 / 1000$ yrs (a) and $1 / 3000$ yrs (b), compared to CALS10k.1b (red) and the VADM reconstruction by Knudsen et al. (2008) (black).

carbon cycle model is not well calibrated to investigate ${ }^{14} \mathrm{C}$ changes on very long time scales. However, different carbon models perform very similarly in terms of modelling the atmospheric ${ }^{14} \mathrm{C}$ concentration (Delaygue \& Bard 2010). Keeping frequencies up to $1 / 1000$ yrs (Fig. 5a) in the radionuclide reconstructions reveals stronger variations, that are rather consistent in the ${ }^{14} \mathrm{C}$ and ${ }^{10} \mathrm{Be}$ record for most of the time except for the interval 4000 to $500 \mathrm{BC}$. Similarities in these variations to the reconstructions based on geomagnetic data hardly seem to exist. Therefore, these variations are likely dominated by solar influence, as e.g. known for the last 500 years where a series of solar minima leads to erroneous low dipole field reconstructions using the radionuclide data.

The geomagnetic reconstructions shown in Figure 5 are also smoothed by their modelling methods, but with different filter characteristics than the radionuclide curves. Particularly, uncertain time scales of the records underlying the geomagnetic field models can lead to a dampening of higher-frequency changes. We used the higher resolution CALS3k.3 and CALS3k.4 dipole estimates for comparisons using the same filters on the geomagnetic and the radionuclide dipole reconstructions for the past 3 kyrs (Fig. 6), and computed the correlation coefficients among all four records for the time series with low-pass filters between $1 / 200$ and 1/1000 yrs. The figure shows that the agreement between the two geomagnetic or the two radionuclide reconstructions, respectively, is better than between the results from the different methods. The correlation coefficient between CALS3k.3 and CALS3k.4 lies in the order of 0.7, and similar values are obtained for the correlation coefficients between ${ }^{14} \mathrm{C}$ and ${ }^{10} \mathrm{Be}$. As seen in Figure 6, particularly the higher-frequency variations in the two radionuclide estimates agree rather well in phase and show higher amplitudes than the geomagnetic reconstructions, confirming the results by Snowball et al. (2007) that variations in radionuclide production rates on up to multicentennial time scales are dominated by solar magnetic field variations. Correlations between geomagnetic and radionuclide reconstructions are lower in nearly all cases. However, especially panels $\mathrm{b}$ and $\mathrm{c}$ in Figure 6 indicate that some common variability is present in the radionuclide data and the CALS3k models on time scales of 500 years and longer (e.g., minimum around $700 \mathrm{AD}$ in Fig. 6b). The correlation coefficients 

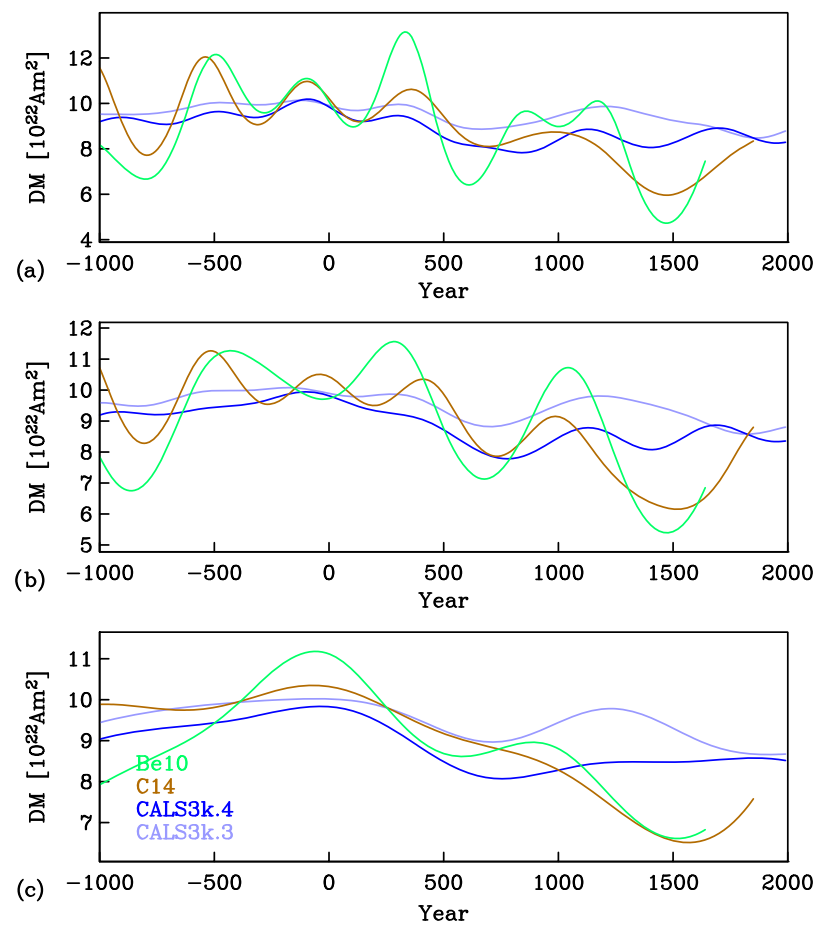

Fig. 6. Dipole moment reconstructions from geomagnetic models CALS3k.3 (light blue) and CALS3k.4 (dark blue) and based on ${ }^{14} \mathrm{C}$ (brown) and ${ }^{10} \mathrm{Be}$ (green) records. The radionuclide-based reconstructions are normalised that they exhibit the same average intensity as suggested by the CALS3k.4 model. All reconstructions are lowpass filtered with cut-off frequency $1 / 400$ yrs (a), 1/500 yrs (b) and $1 / 1000$ yrs (c).

between CALS3k.3 and ${ }^{10} \mathrm{Be}$ or CALS3k.4 and the radionuclide records mostly lie in the order of $0.4-0.5$, probably on the verge of statistical significance for these filtered, serially correlated, time series. The correlation coefficients between CALS3k.3 and the ${ }^{14} \mathrm{C}$-based estimate reach values no higher than 0.1 and certainly cannot be considered significant. However, the fact that the correlation of the radionuclide records with CALS3k.4 is consistently higher for all filter frequencies than with CALS3k.3 could indicate that the more recent geomagnetic model version indeed provides an improved dipole moment variability estimate. The highest correlation between the different method reconstructions is obtained between CALS3k.4 and ${ }^{14} \mathrm{C}$ dipole moments with a low-pass filter of $1 / 1000$ yrs. It reaches 0.7 and suggests that a significant portion of the variability is common to both records. As seen in Fig. 6c, there is a very good agreement between these two estimates for the time interval $1000 \mathrm{BC}$ to $750 \mathrm{AD}$. Although the ${ }^{10} \mathrm{Be}$-based record shows larger variability, the variations are still in phase. If not incidental, it contradicts our earlier result from the longerterm comparison, where we concluded that variations on the order of a millennium are not in agreement between the radionuclide records and the output of the geomagnetic field models. This emphasises again the need for a better understanding of multi-centennial range dipole variations based on geomagnetic data and it could suggest that some higher-frequency variability gets lost when compiling a large number of records with uncertain time scales.

\section{Conclusions}

We have reviewed and discussed several recent geomagnetic field and dipole reconstructions of the past 3-10 millennia.
Uncertainties in the models are far from small, mainly due to an extremely inhomogeneous data distribution and inherently large data and dating uncertainties in large parts of the data set, which remain despite the progress and huge efforts made by archeo- and paleomagnetists. It is difficult to fully assess the reliability of the different aspects of the individual reconstructions. A comparison of models obtained by different methods can help to evaluate which features appear robust and which require further investigation.

Different dipole axis reconstructions appear to converge at least for the recent three millennia, but a notable discrepancy remains among dipole moment estimates, where VADMs give consistently higher values than spherical harmonic models. Reasons for both over- and underestimations are conceivable and although there are indications that VADMs might tend to overestimate the dipole moment during recent millennia, only a considerably improved data distribution, particularly regarding archeomagnetic intensity data from the southern hemisphere, will fully resolve the inconsistency. Regarding the family of spherical harmonic models, CALS10k.1b is substantially improved compared to the previous CALS7K.2, which suffered from a lack of intensity information prior to about 1000 BC. For the recent 3 kyrs, CALS3k. 4 should be the best choice, although its comparatively low dipole moment estimate is surprising.

Cosmogenic radionuclide production rates can provide independent estimates of past dipole intensity changes, but it is not possible to accurately separate geomagnetic from solar modulation. Our results confirm that the solar influence dominates the short periods and geomagnetic changes probably start to play a significant role on time scales from 500 yrs and longer. However, they also suggest that there still is a notable solar variation influence on time scales up to a few millennia. The somewhat contradictory results for the similarity of radionuclide-based dipole reconstructions and geomagnetic field models on these time scales point out that geomagnetic field models have to be improved further before they can provide a truly robust means to eliminate the influence of geomagnetic variability in cosmogenic radionuclide production studies. At present it seems advisable to consider if the choice of geomagnetic field model would make a significant difference to any interpretation.

Continuing efforts in several directions are required to improve our knowledge about past magnetic field changes. Improved modelling techniques and comparisons of refined future models can bring some progress. Further advances in understanding the acquisition of magnetisation in archeo- and paleomagnetic data, continuing development of improved laboratory procedures and further investigations of suitability and appropriate scaling of sedimentary relative intensity records will help to reduce uncertainties in the data, and consequently also in the models. Improvements to the dating of archeomagnetic samples and particularly sediment records would be particularly useful. The most essential requirement, however, is new data from as yet sparsely covered regions.

\section{References}

Delaygue, G., and E. Bard, An Antarctic view of Beryllium-10 and solar activity for the past millennium, Clim. Dyn., 36, 2201-2218, 2010.

Donadini, F., K. Korhonen, P. Riisager, and L.J. Pesonen, Database for Holocene geomagnetic intensity information, EOS Trans. Am. Geophys. Soc., 87 (14), 92-93, 2006. 
Donadini, F., M. Korte, and C. Constable, Geomagnetic field for 0-3ka: 1. new data sets for global modeling, Geochem. Geophys. Geosyst., 10, Q06007, DOI: 10.1029/2008GC002295, 2009.

Donadini, F., M. Korte, and C. Constable, Millennial variations of the geomagnetic field: from data recovery to field reconstruction, Space Sci. Rev., 155, 219-246, 2010.

Finkel, R. C., and K. Nishiizumi, Beryllium 10 concentrations in the Greenland Ice Sheet Project 2 ice core from 3-40 ka, J. Geophys. Res., 102, 26699-26706, 1997.

Genevey, A., Y. Gallet, C. Constable, M. Korte, and G. Hulot, ArcheoInt: An upgraded compilation of geomagnetic field intensity data for the past ten millennia and its application to the recovery of the past dipole moment, Geochem. Geophys. Geosyst., 9, Q04038, DOI: 10.1029/2007GC001881, 2008.

Heirtzler, J., The future of the south atlantic anomaly and implications for radiation damage in space, J. Atmos. Sol. Terr. Phys., 64, 1701-1708, 2002.

Hulot, G., A. Khokhlov, and J. L. LeMouël, Uniqueness of mainly dipolar magnetic fields recovered from directional data, Geophys. J. Int., 129, 347-354, 1997.

Jackson, A., A. R. T. Jonkers, and M. R. Walker, Four centuries of geomagnetic secular variation from historical records, Philos. Trans. R. Soc. London, Ser. A, 358, 957-990, 2000.

Knudsen, M. F., P. Riisager, F. Donadini, I. Snowball, R. Muscheler, K. Korhonen, L. J. Pesonen, and B. H. Jacobsen, Variations in the geomagnetic dipole moment during the Holocene and the past 50 kyr, Earth Planet. Sci. Lett., 272, 319-329, 2008.

Korhonen, K., F. Donadini, P. Riisager, and L.J. Pesonen, Geomagia50: an archeointensity database with PHP and MySQL, Geochem. Geophys. Geosyst., 9, Q04029, DOI: 10.1029/2007GC001893, 2008

Korte, M., and C.G. Constable, Continuous geomagnetic field models for the past 7 millennia: 2. CALS7K, Geochem. Geophys. Geosyst., 6, Q02H16, DOI: 10.1029/2004GC000801, 2005a.

Korte, M., and C. G. Constable, The geomagnetic dipole moment over the last 7000 years - new results from a global model, Earth Planet. Sci. Lett., 236, 348-358, 2005b.

Korte, M., and C. Constable, Improving geomagnetic field reconstructions for 0-3 ka, Phys. Earth Planet. Inter., 188, 247-259, 2011

Korte, M., C. Constable, F. Donadini, and R. Holme, Reconstructing the Holocene geomagnetic field, Earth Planet. Sci. Lett., 312, 497-505, 2011.

Korte, M., F. Donadini, and C. Constable, Geomagnetic field for 0-3ka: 2. a new series of time-varying global models, Geochem. Geophys. Geosyst., 10, Q06008, DOI: 10.1029/2008GC002297, 2009.

Korte, M., A. Genevey, C.G. Constable, U. Frank, and E. Schnepp, Continuous geomagnetic field models for the past 7 millennia: 1 . A new global data compilation, Geochem. Geophys. Geosyst., 6, Q02H15, DOI: 10.1029/2004GC000800, 2005.

Lifton, N., D. F. Smart, and M. A. Shea, Scaling time-integrated in situ cosmogenic nuclide production rates using a continuous geomagnetic model, Earth Planet. Sci. Lett., 268, 190-201, 2008.

Marsh, N., and H. Svensmark, Solar influence on earth's climate, Space Sci. Rev., 107, 317-325, 2003.

Masarik, J., and J. Beer, Simulation of particle fluxes and cosmogenic nuclide production in the Earth's atmosphere, J. Geophys. Res., 104 (12), 12099-12111, 1999.

Muscheler, R., J. Beer, P. Kubik, and H.-A. Synal, Geomagnetic field intensity during the last 60,000 years based on $10 \mathrm{Be} \& 36 \mathrm{Cl}$ from the Summit ice cores and 14C, Quat. Sci. Rev., 24, 1849-1860, 2005a.

Muscheler, R., J. Beer, G. Wagner, C. Laj, C. Kissel, G. M. Raisbeck, F. Yiou, and P. W. Kubik, Changes in the carbon cycle during the last deglaciation as indicated by the comparison of $10 \mathrm{Be}$ and $14 \mathrm{C}$ records, Earth Planet. Sci. Lett., 219, 325-340, 2004.

Muscheler, R., and U. Heikkilä, Constraints on long-term changes in solar activity from the range of variability of cosmogenic radionuclide records, Astrophys. Space Sci. Trans., 7, 355-364, 2011.

Muscheler, R., F. Joos, S. A. Muller, and I. Snowball, How unusual is today's solar activity?, Nature, 436, E3-E4, $2005 \mathrm{~b}$.

Nilsson, A., R. Muscheler, and I. Snowball, Millennial scale cyclicity in the geodynamo inferred from a dipole tilt reconstruction, Earth Planet. Sci. Lett., 311, 299-305, 2011.

Nilsson, A., I. Snowball, and R. Muscheler, Holocene geocentric dipole tilt model constrained by sedimentary paleomagnetic data, Geochem. Geophys. Geosyst., 11, Q08018, DOI: 10.1029/2010GC003118, 2010.

Pigati, J. S., and N. A. Lifton, Geomagnetic effects on timeintegrated cosmogenic nuclide production with emphasis on in situ ${ }^{14} \mathrm{C}$ and ${ }^{10} \mathrm{Be}$, Earth Planet. Sci. Lett., 226, 193-205, 2004.

Rasmussen, S. O., K. K. Andersen, A. M. Svensson, J. P. Steffensen, B. M. Vinther, et al., A new Greenland ice core chronology for the last glacial termination, J. Geophys. Res., 111, D06102, DOI: 10.1029/2005JD006079, 2006.

Reimer, P. J., M. G. L. Baillie, E. Bard, A. Bayliss, J. W. Beck, et al., IntCal04 Terrestrial radiocarbon age calibration, 26-0 ka BP, Radiocarbon, 46, 1029-1058, 2004

Snowball, I., and R. Muscheler, Palaeomagnetic intensity data: an Achilles heel of solar activity reconstructions, The Holocene, 17 (6), 851-859, 2007.

Snowball, I., L. Zillén, A. Ojala, T. Saarinen, and P. Sandgren, FENNOSTACK and FENNORPIS: Varve dated Holocene palaeomagnetic secular variation and relative palaeointensity stacks for Fennoscandia, Earth Planet. Sci. Lett., 255, 106-116, 2007.

Solanki, S., I. Usoskin, B. Kromer, M. Schussler, and J. Beer, Unusual activity of the sun during the recent decades compared to the previous 11,000 years, Nature, 431, 1084-1087, 2004.

Usoskin, I., K. Horiuchi, S. Solanki, G. A. Kovaltsov, and E. Bard, On the common solar signal in different cosmogenic isotope data sets, J. Geophys. Res., 114, A03112, DOI: $10.1029 / 2008 J A 013888,2009$.

Usoskin, I., M. Korte, and G. Kovaltsov, Role of centennial geomagnetic changes in local atmospheric ionization, Geophys. Res. Lett., 35, L05811, DOI: 10.1029/2007GL033040, 2008.

Usoskin, I. G., I. A. Mironova, M. Korte, and G. A. Kovaltsov, Regional millennial trend in the cosmic ray induced ionization of the troposphere, J. Atm. Sol. Terr. Phys., 72, 19-25, 2010.

Usoskin, I. G., S. K. Solanki, and M. Korte, Solar activity reconstructed over the last 7000 years: the influence of geomagnetic field changes, Geophys. Res. Lett., 33, L08103, DOI: 10.1029/2006GL025921, 2006.

Valet, J.-P., E. Herrero-Bervera, J.-L. LeMouël, and G. Plenier, Secular variation of the geomagnetic dipole during the past 2000 years, Geochem. Geophys. Geosyst., 9, Q01008, DOI: 10.1029/2007GC001728, 2008.

Vonmoos, M., J. Beer, and R. Muscheler, Large variations in Holocene solar activity: constraints from $10 \mathrm{Be}$ in the Greenland Ice Core Project ice core, J. Geophys. Res., 111, A10105, DOI: 10.1029/2005JA011500, 2006. 